\title{
BMJ Open Diagnosing Potentially Preventable Hospitalisations (DaPPHne): protocol for a mixed-methods data-linkage study
}

\author{
Megan E Passey, ${ }^{1}$ Jo M Longman, ${ }^{1}$ Jennifer J Johnston, ${ }^{1}$ Louisa Jorm, ${ }^{2}$ \\ Dan Ewald, ${ }^{3}$ Geoff G Morgan, ${ }^{1}$ Margaret Rolfe, ${ }^{1}$ Bronwyn Chalker ${ }^{4}$
}

To cite: Passey ME, Longman JM, Johnston JJ, et al. Diagnosing Potentially Preventable Hospitalisations (DaPPHne): protocol for a mixed-methods data-linkage study. BMJ Open 2015;5: e009879. doi:10.1136/ bmjopen-2015-009879

- Prepublication history for this paper is available online To view these files please visit the journal online (http://dx.doi.org/10.1136/ bmjopen-2015-009879).

Received 1 September 2015 Revised 16 October 2015 Accepted 27 October 2015

CrossMark

${ }^{1}$ University Centre for Rural Health-North Coast, University of Sydney, Lismore, New South Wales, Australia

${ }^{2}$ Centre for Big Data Research in Health, University of New South Wales, Sydney, New South Wales, Australia ${ }^{3}$ North Coast Primary Health Network, Ballina, New South Wales, Australia

${ }^{4}$ Mid North Coast Local Health District, South West Rocks, New South Wales, Australia

Correspondence to Dr Megan Passey; megan.passey@ucrh.edu.au

\section{ABSTRACT}

Introduction: Rates of potentially preventable hospitalisations (PPH) are used as a proxy measure of effectiveness of, or access to community-based health services. The validity of PPH as an indicator in Australia has not been confirmed. Available evidence suggests that patient-related, clinician-related and systems-related factors are associated with PPH, with differences between rural and metropolitan settings. Furthermore, the proportion of PPHs which are actually preventable is unknown. The Diagnosing Potentially Preventable Hospitalisations study will determine the proportion of PPHs for chronic conditions that are deemed preventable and identify potentially modifiable factors driving these, in order to develop effective interventions to reduce admissions and improve measures of health system performance.

Methods and analysis: This mixed methods data linkage study of approximately 1000 eligible patients with chronic PPH admissions to one metropolitan and two regional hospitals over 12 months will combine data from multiple sources to assess the: extent of preventability of chronic PPH admissions; validity of the Preventability Assessment Tool (PAT) in identifying preventable admissions; factors contributing to chronic PPH admissions. Data collected from patients (quantitative and qualitative methods), their general practitioners, hospital clinicians and hospital records, will be linked with routinely collected New South Wales (NSW) Admitted Patient Data Collection, the NSW Registry of Births, Death and Marriages death registration and Australian Bureau of Statistics mortality data. The validity of the PAT will be assessed by determining concordance between clinician assessment and that of a 'gold standard' panel. Multivariable logistic regression will identify the main predictor variables of admissions deemed preventable, using study-specific and linked data.

Ethics and dissemination: The NSW Population and Health Services Research Ethics Committee granted ethical approval. Dissemination mechanisms include engagement of policy stakeholders through a project Steering Committee, and the production of summary reports for policy and clinical audiences in addition to peer-review papers.

\section{INTRODUCTION}

Potentially preventable hospitalisations ( $\mathrm{PPH}$; also referred to as admissions for ambulatory care sensitive conditions) are admissions considered to be potentially preventable with effective, timely outpatient care in the period immediately prior to admission. ${ }^{1}$ The concept of $\mathrm{PPH}$ admissions originated in New York in the early $1990 \mathrm{~s}^{1}$ and since then has been widely used as an indicator of effectiveness of, or access to, primary healthcare in many countries. In Australia, the rate of $\mathrm{PPH}$ is a key performance indicator (KPI) in the National Healthcare Agreement (NHA) ${ }^{2}$ and the National Health Performance and Accountability Framework ${ }^{3}$ and is therefore tied directly to hospital funding.

PPH conditions are categorised as vaccinepreventable, acute or chronic, with the specific conditions classified as PPH varying across countries. According to the NHA definition, ${ }^{2}$ there were more than $772000 \mathrm{PPH}$ admissions in Australia in 2012-2013, accounting for $10.3 \%$ of all public hospital admissions, with higher rates in remote and very remote areas. ${ }^{4}$ Over half of Australian PPH admissions are attributable to chronic conditions, with congestive cardiac failure (CCF), chronic obstructive pulmonary disease (COPD), diabetes complications and angina accounting for over $97 \%$ of all chronic PPH admissions. ${ }^{4}$

Despite its use, however, the validity of PPH as an indicator of effectiveness or access to care has not been definitively confirmed in the Australian setting. ${ }^{5}$ Although one Australian study found that better self-rated access to care was associated with lower rates of PPH in urban areas, this was not the case in rural areas. ${ }^{6}$ Furthermore, a number of socioeconomic and behavioural factors were associated with $\mathrm{PPH},{ }^{6}$ leading the authors to suggest that the association between healthcare effectiveness and access and $\mathrm{PPH}$ is complex, requiring further research. ${ }^{6}$ Preliminary work undertaken by the current research team confirmed this complexity. ${ }^{7-9}$ Indeed, a recent study found personal 
sociodemographic and health characteristics, rather than general practitioner (GP) supply, are major drivers for PPH in Australia, particularly for chronic conditions. $^{10}$

In an attempt to reduce unnecessary admissions to hospital, and achieve KPI targets related to PPH admissions, policymakers and health services have developed and implemented programmes specifically targeting patients with $\mathrm{PPH}$ admissions, such as the New South Wales (NSW) Chronic Disease Management Program, with the aim of improving the coordination of their care. ${ }^{11}$ In doing this, a population-level indicator is now being used at the individual level to identify patients who may benefit from additional support. However, this response to $\mathrm{PPH}$ admissions is significantly limited by the fact that the proportion of PPH admissions that is actually preventable is unknown and there is no easy way for a health system or researchers to identify which individual admissions are actually preventable. PPH are identified on the basis of diagnostic codes in hospital administrative data. ${ }^{12-15}$ While this approach takes advantage of the availability of administrative data sets, it overestimates rates of preventable admissions because it also captures an unknown number of admissions that are necessary and could not feasibly have been prevented. Few studies have attempted to assess preventability of individual admissions, and almost all of these have focused on readmissions. A recent systematic review of the preventability of readmissions reported a median proportion of $27.1 \%$ as actually preventable, however estimates ranged from $5 \%$ to $79 \% .{ }^{16}$ No such studies have been undertaken in Australia.

Very little is known about patients' perspectives of the underlying factors contributing to individual PPH admissions. ${ }^{17}$ Understanding patients' views on what influenced their decision to go to hospital, the support they received in the lead-up to their admission, and what may have helped to prevent the admission, may help identify leverage points and mechanisms for reducing PPH admissions.

In order to develop and target effective interventions to reduce PPHs, and to inform the appropriate use of PPH measures as indicators of health system performance, we need to identify the proportion of PPH admissions that are considered preventable and identify the drivers of these preventable admissions.

The DaPPHne project aims to do this. Its specific objectives are to:

1. Validate a tool for use by clinicians and researchers to assess the preventability of individual admissions;

2. Determine the proportion of chronic PPH admissions among community-dwelling patients $\geq 45$ years that is deemed to be preventable;

3. Identify factors contributing to PPH admissions classified as preventable;

4. Recommend refinements to PPH measures that can be applied nationally and internationally to provide more robust health service performance measurement based on admissions deemed to be preventable
5. Identify interventions to reduce chronic $\mathrm{PPH}$ admissions.

\section{Definition of preventability}

In this study, we use the definition of a preventable admission provided in the box below. We were unable to identify any clear timeframes for preventability in the PPH literature, although the original work by Billings et $a l^{1}$ referred to the "period immediately prior to admission'. Based on our understanding of the literature, and in consultation with clinicians and other researchers in the field, we determined that a 3-month time frame was reasonable and that the definition should include access to and utilisation of health and social services, as well as patient health behaviours.

A preventable admission is defined as an unplanned admission which could have been prevented if:

1. Appropriate, adequate, accessible and good quality support in the community* had been available and accessed in the preceding 3 months, and/or

2. Appropriate individual health behaviours, for example, disease self-management, had occurred in the 3 months prior to admission.

*Support in the community might include primary healthcare, family/neighbour/friend/social support, health or non-health community services.

\section{METHODS AND ANALYSIS}

This mixed methods data linkage study of approximately 1000 chronic PPH admissions will combine a wide range of study-specific and routinely collected data to enable exploration of the factors contributing to chronic PPH admissions. One metropolitan and two regional hospitals will participate.

The substudies to be undertaken are:

1. Comprehensive data collection for each admission to identify factors associated with preventable admissions including data collected from the patient, hospital records and the patients' GP (if available). Two senior hospital clinicians will complete an assessment of the preventability of each admission. Admissions deemed preventable will be compared with those deemed not preventable to identify patient, clinician and system factors associated with preventable admissions.

2. Validation of a Preventability Assessment Tool (PAT): For the first 150 admissions with complete data, the assessment by hospital clinicians using a PAT that we have previously developed and piloted ${ }^{18}$ will be compared against a 'gold standard'-an assessment by a panel of senior clinicians, using a modified version of the process developed by Oddone $e t a l .{ }^{19}$ This will be done separately for the metropolitan and the two regional hospitals (ie, 150 admissions for each) to allow assessment of validity in both settings due to 
the different service characteristics and demographics of the catchment populations. The metropolitan hospital serves a younger and more ethnically diverse population, with a greater proportion of people who do not speak English at home.

3. Qualitative study: A subset of approximately 20 consenting patients will participate in semistructured interviews to elicit their perspectives of the circumstances leading up to their admission, and whether they can identify any measures that may have prevented their hospital admission. Interviews will be analysed thematically.

4. Predicting preventability using routinely collected data: Study-specific data will be linked to administrative data relating to hospital admissions and deaths. These linked data will be used to develop models to predict the preventability of individual admissions using routine administrative data and investigate how additional items (eg, measures of self-rated health) can improve the prediction of preventability.

The contribution of each substudy to the study objectives is shown in table 1.

\section{Recruitment}

Research nurses will be employed at each hospital to facilitate participant recruitment and data collection. Through close liaison with emergency department (ED) staff, the research nurses will identify all patients presenting to the ED who potentially meet the eligibility criteria (see below). Once a decision has been made to admit a patient, the research nurse will confirm eligibility and invite them to participate. Patients will provide written consent to all aspects of the study, with the option of declining individual substudies. To minimise bias in selection of patients, while accommodating the extensive data collection requirements, patients will be recruited every second week, and all eligible patients will be invited during recruitment weeks, regardless of the time of day or day of week, or whether they have previously participated in the study. During the alternate (nonrecruitment) weeks, the research nurse will complete data collection for patients recruited the previous week (ie, hospital clinical data, GP data). As there is considerable seasonal variation in admission rates for these conditions, patients will be recruited over a 12-month period.

\section{Sample eligibility criteria}

Eligible patients are community-dwelling adults aged $\geq 45$ years with an unplanned admission to any of the participating hospitals with a primary diagnosis of selected chronic PPH conditions (CCF, COPD, diabetes complications and angina as defined by the Australian Institute of Health and Welfare ${ }^{20}$-see table 2). Exclusion criteria are cognitive impairment such that the patient is unable to give informed consent; patient living in residential aged care facility, prison or other facility; final discharge diagnosis not one of the specified inclusion diagnoses; and transfer from another hospital.

\section{Data sources and collection}

Data will be collected regarding each eligible and consenting admission via patient questionnaire, the PAT, GP interview and extraction of hospital clinical data, as described below.

\section{Patient questionnaire}

The research nurse will administer a study-specific questionnaire, taking approximately $30 \mathrm{~min}$ to complete. Information will be collected regarding sociodemographic characteristics, health status (SF-36v2 ${ }^{21}$ ); psychological distress (Kessler $10^{22}$ ); disease self-management (Partners in Health Scale ${ }^{23}$ ); health literacy (REALM-R $^{24}$ and Chew $^{25}$ ); lifestyle risk factors; social support (abbreviated Duke Social Support Index ${ }^{26}$ and subscales of the ENRICHED Social Support Inventory ${ }^{27}$ ); medications adherence and complications (Morisky 8-item Medication Adherence questionnaire ${ }^{28}$

Table 1 Relationship between project objectives and substudies

\begin{tabular}{|c|c|c|c|c|}
\hline & $\begin{array}{l}\text { Substudy 1: comprehensive } \\
\text { data collection for each } \\
\text { admission }\end{array}$ & $\begin{array}{l}\text { Substudy 2: } \\
\text { validation of the } \\
\text { PAT }\end{array}$ & $\begin{array}{l}\text { Substudy } 3: \\
\text { qualitative study }\end{array}$ & $\begin{array}{l}\text { Substudy 4: } \\
\text { data linkage }\end{array}$ \\
\hline Objective 1: validate PAT & $\mathrm{X}$ & $x$ & & \\
\hline $\begin{array}{l}\text { Objective 2: assess proportion } \\
\text { of PPH admissions deemed } \\
\text { preventable }\end{array}$ & $\mathrm{X}$ & $x$ & & \\
\hline $\begin{array}{l}\text { Objective } 3 \text { : identify factors } \\
\text { contributing to preventable } \\
\text { hospitalisations }\end{array}$ & $x$ & & $\mathrm{x}$ & $x$ \\
\hline $\begin{array}{l}\text { Objective } 4 \text { : recommend } \\
\text { refinements to } \mathrm{PPH} \text { measures }\end{array}$ & $x$ & & & $x$ \\
\hline $\begin{array}{l}\text { Objective } 5 \text { : identify } \\
\text { interventions to reduce chronic } \\
\text { PPH admissions }\end{array}$ & $x$ & & $\mathrm{x}$ & $x$ \\
\hline
\end{tabular}


150

150.0

150.1

I 50.9

I 11.0

J 81

120

I 20.0

I 20.1

I 20.8

120.9

I 24.0

124.8

124.9

J 41

$\mathrm{J} 42$

J 43

$\mathrm{J} 44$

J 47

J 20

E 10

E 11

E 12

E 13

E 14

E 87.0

E 87.2

G 45

G $50-$ G 64

$\mathrm{H} 25-\mathrm{H} 28$

$\mathrm{H} 30-\mathrm{H} 36$

$\mathrm{H} 40$ \& $\mathrm{H} 42$

120

| $21-122$

| $23-125$

125

150

I 60-1 64, I 69

$170-174$

$\mathrm{K} 05$

N 00-N 29

Z 49
Congestive cardiac failure

Any of the following as principal diagnosis only

Heart failure

Congestive heart failure

Left ventricular failure

Heart failure, unspecified

Hypertensive heart disease with (congestive) heart failure

Pulmonary oedema

Angina

Any of the following as principal diagnosis only

Angina pectoris

Unstable angina

Angina pectoris with documented spasm

Other forms of angina pectoris

Angina pectoris, unspecified

Coronary thrombosis not resulting in myocardial infarction

Other forms of acute ischaemic heart disease

Acute ischaemic heart disease, unspecified

Chronic obstructive pulmonary disease

Any of the following as principal diagnosis only

Simple and mucopurulent chronic bronchitis

Unspecified chronic bronchitis

Emphysema

Other chronic obstructive pulmonary disease

Bronchiectasis

$\mathrm{J} 20$ as principal diagnosis ONLY if additional diagnoses of $\mathrm{J} 41, \mathrm{~J} 42$,

$\mathrm{J} 43, \mathrm{~J} 44, \mathrm{~J} 47$

Acute bronchitis

Diabetes and diabetes complications

Any of the following as principal diagnosis only

Type 1 diabetes mellitus with or without complications

Type 2 diabetes mellitus with or without complications

Malnutrition-related diabetes mellitus

Other specified diabetes mellitus

Unspecified diabetes mellitus

E 10-E 14 as additional diagnoses where the

principal diagnosis is one of

Hyperosmolarity

Acidosis

Transient ischaemic attack

Nerve disorders and neuropathies

Cataracts and lens disorders

Retinal disorders

Glaucoma (all)

Angina pectoris

Myocardial infarction

Other acute and chronic ischaemic heart diseases

Chronic ischaemic heart disease

Heart failure

Stroke and sequelae

Peripheral vascular disease

Gingivitis and periodontal diseases

Kidney diseases including end-stage renal disease

Renal dialysis and items from the Pit Medication Risk Assessment Form ${ }^{29}$ ), and access and barriers to healthcare including contact with GPs, specialists and allied health professionals in the previous 12 months.
Preventability Assessment Tool

Given the importance of having a method to assess the preventability of individual $\mathrm{PPH}$ admissions, the PAT was developed for use by senior hospital clinicians to assess 
the preventability of individual chronic PPH admissions. The tool, based on an extensive literature review and consultation with clinicians, draws on the earlier work of Oddone $e t a l^{19}$ and Arozullah $e t a \hat{l}^{30}$ who assessed preventability of general medical readmissions and admissions using a retrospective audit process. Our tool can be used by clinicians during the admission, rather than retrospectively, and is designed specifically for $\mathrm{PPH}$ admissions. It also considers individual and social factors more extensively than the earlier work and defines the timeframe for preventability as the previous 3 months.

Clinicians are asked to indicate the reason for admission and to rate the extent to which they consider that a range of patient, clinician and system factors contributed to that admission (using a scale of 1-4). The tool concludes with a global assessment of the preventability of the admission (given currently available services and social support) on a scale of $1-10$, action that could have been taken to prevent the admission, and asks for suggestions for improved/additional services/social support which could have helped prevent the admission. The PAT has had face and content validity confirmed and was assessed in a small pilot study in two hospitals. ${ }^{18}$ It takes about 5 min to complete.

For each admission, the research nurses will provide copies of the PAT to the medical registrar and a senior nurse caring for the patient and facilitate form completion.

\section{Structured GP telephone interview}

Patients' GPs will be interviewed regarding: care provided; practice factors; adherence to selected elements of the guidelines for management of patients' PPH diagnoses; other chronic conditions (including mental health) and/or social issues with the potential to impact on patients' self-management; and use of out-of-hospital clinical and non-clinical services. Finally, GPs will be asked to consider whether, assuming available services and social support, the admission was preventable (as defined above) and if so, what action could have been taken to prevent the admission. GPs will also be asked for suggestions for improved and/or additional services and/or social support which could have helped prevent the admission. The interview will take 15-20 min and GPs will be reimbursed for their time.

\section{Hospital clinical data}

The research nurses will extract clinical data from the hospital records for the participants' current admission and the most recent previous admission. Items extracted from the patients' notes will include reasons for presentation to hospital, principal diagnoses and comorbidities, medications on admission, and discharge information. For admissions included in the validation of the PAT, clinical notes for the first $24 \mathrm{~h}$ and the hospital discharge summary will also be obtained to assist the expert panel involved in the PAT validation (similar to the study by Oddone $e t a l^{19}$ ).

\section{Qualitative study}

Participants who have consented to participate in the qualitative study will be contacted for an interview. Purposive sampling will be used to ensure a range of sex, age, condition, location and a majority of preventable admissions (as determined by the PAT), in order to ensure sufficient data regarding preventable admissions. Sampling will cease when saturation of themes is reached.

This substudy will use semistructured interviews to elicit patient perspectives. Interviews will explore the circumstances around the admission on the day of admission (including why and how they made the decision to seek help), their state of health and any changes to it in the 3 months leading up to the admission; their home/ social and health services support (and any changes to this support) in the 3 months leading up to the admission, and whether they can identify anything that may have helped to prevent the admission. An interview guide will be used to focus the discussion. Interviews will be recorded and transcribed verbatim.

\section{Data linkage}

Data collected through the PAT, patient and GP questionnaires will be linked to the NSW Admitted Patient Data Collection (APDC), which includes records for all separations from all NSW public and private hospitals and NSW Registry of Births, Death and Marriages (RBDM) death registration (fact-of-death) and ABS mortality data for the period February 2009 to December 2016 (end of recruitment).

The Centre for Health Record Linkage (CHeReL) will perform the linkage, using probabilistic record linkage techniques and ChoiceMaker software. ${ }^{31}$ Quality assurance data show false-positive and false-negative rates of around $0.5 \% .^{32}$

\section{Analysis plan}

Substudy 1: Identifying factors associated with preventable admissions

Analysis will be performed using STATA V.9.0. Univariate comparisons of patient characteristics, clinical problems, use of services and care provided will be undertaken using standard statistical tests $\left(\chi^{2}\right.$ test for categorical variables and t tests or non-parametric equivalents for continuous variables). The primary outcome for assessment of preventability will be the judgement of the hospital clinicians using the PAT. The choice of whether the assessments of the physicians or senior nurses, or a combination, is used will depend on the outcome of the validation of the tool (see Substudy 2 below).

Multivariable logistic regression will be performed to identify the main predictor variables for preventable admissions as determined by the PAT (outcome variable, coded as $0=$ not preventable, $1=$ preventable). Initially all variables with $p<0.25$ in the univariate analyses will be included as predictor variables in the model. The influence of each variable will be assessed using Wald tests, 
with stepwise removal of variables with $p \geq 0.05$. Age and gender will be retained in the model regardless of significance. The possible clustering by site will be addressed by including site as a fixed variable in the model, and assessing interaction effects. It is possible that some patients will have multiple admissions and this will be explored using generalised estimating equations to adjust for clustering. The analysis will identify factors amenable to interventions as well as patient characteristics associated with preventable admissions.

For those admissions deemed preventable, the gaps in services and other factors that the clinicians (ie, GPs and senior hospital clinicians) or patient identify as contributing to this admission will be coded and summarised using descriptive statistics and qualitative analysis. Variables for these analyses will be derived from the GP interview, the PAT and the patient questionnaire.

\section{Substudy 2: Validation of the PAT}

The judgement of an expert panel, consisting of a hospital physician, GP and community nurse with expertise in chronic disease management, will be used as the 'gold standard' for validation of the PAT. The data collected in the patient questionnaire, hospital clinical data (including a copy of all clinical data from the first $24 \mathrm{~h}$ of admission), the hospital discharge summary and structured GP interview will be consolidated into a nonidentifiable 'case summary' for review by the expert panel. Following training, each member of the panel will review each summary, blinded to the assessments made by the hospital clinicians or each other, and provide an assessment of whether they were 'reasonably confident that this admission was preventable' (yes/no). For those classified as 'preventable', panel members will identify interventions they consider could have prevented the admission. When discrepancies in the assessment of preventability occur between the panel members, a meeting of the panel will be convened to discuss the case and come to consensus. The process is based on that developed by Oddone et al, ${ }^{19}$ but with the following modifications: more information is provided to the panel to make their assessment, including data from the GP and the patient; and the panel includes a wider range of clinicians, thereby bringing different perspectives to the assessment.

Concordance between the assessment of preventability made by each of the hospital clinicians using the PAT, and the assessment of the expert panel will be assessed separately (ie, physician and senior nurse separately) and in combination (ie, both assess as preventable, neither assess as preventable), using the $\kappa$ statistic, sensitivity and specificity. The optimal cut-off score for the PAT scale will be determined post hoc.

Following validation of the PAT, this tool will be used to assess preventability of all study admissions in Substudy 1 .

\section{Substudy 3: Qualitative study}

Interviews will be audio recorded, with the recordings professionally transcribed verbatim and analysed thematically following Braun and Clarke. ${ }^{33}$ These transcripts will be initially coded into broad categories and subcategories and then synthesised into themes. The analysis will take place from the beginning of data collection and continue until saturation of themes is reached.

\section{Substudy 4: Predicting preventability using routinely collected data}

Logistic regression will be used to build predictive models for the preventability of specific admissions, as defined according to the PAT, using stepwise approaches. The predictive variables derived from the APDC will include patient age, sex, remoteness of residence and other demographics, the principal and other diagnoses recorded in the index admission, principal and other procedures, comorbidity indeces, incident versus subsequent admissions, and source of referral. An automated approach to variable selection will be adopted, using the full range of APDC variables, because the purpose of predictive modelling is to create the best model to predict future events using the data available, rather than to test a priori hypotheses regarding the contribution of causal factors. Finally, we will explore whether adding additional items derived from questionnaire data (eg, self-rated health, social isolation and medication adherence) to administrative data results in improvements to the predictive power of the models, by comparing model fit metrics.

Statistical analysis will be performed with SAS V.9.3. ${ }^{34}$ Analysis of linked data will be performed within a dedicated workspace in the Secure Unified Research Environment (SURE, https://www.sure.org.au/) remote access data laboratory.

\section{Statistical power}

\section{Sample size}

Based on admission data it is anticipated there will be approximately 3960 eligible admissions to the participating hospitals over the 12-month recruitment period. The following assumptions are applied to sample size and power calculations: Second weekly recruitment; $50 \%$ consent rates; $15 \%$ loss due to meeting exclusion criteria; $10 \%$ missing data on the PAT and $15 \%$ having missing GP data. Based on these assumptions, we anticipate recruiting 990 patients, with 644 with complete data. The conservative power calculations below assume 600 patients with complete data.

For a power of $80 \%$, and $\alpha=0.05$, table 3 shows the difference detectable for various rates of the factor of interest in the non-preventable group if $15 \%, 25 \%$ and $35 \%$ of admissions are classified as preventable. For example, if $25 \%$ of admissions are classified as preventable, and the factor of interest (eg, no contact with GP in month prior to admission) occurs in $10 \%$ of the nonpreventable group, we will have power to detect a 
Table 3 Difference detectable for various rates of the factor of interest and proportions of admissions classified as preventable

\begin{tabular}{llll}
\hline & \multicolumn{3}{l}{$\begin{array}{l}\text { Proportion classified } \\
\text { as preventable }\end{array}$} \\
\cline { 2 - 4 } $\begin{array}{llll}\text { Rate of factor of interest in } \\
\text { non-preventable groups }\end{array}$ & $\mathbf{1 5 \%}$ & $\mathbf{2 5 \%}$ & $\mathbf{3 5 \%}$ \\
\hline $10 \%$ & Difference detectable \\
$20 \%$ & $11.7 \%$ & $9.5 \%$ & $8.6 \%$ \\
\hline
\end{tabular}

difference in prevalence of $9.5 \%$ between the two groups. With a greater proportion classified as preventable, we are able to detect smaller differences between the groups. Previous studies of preventability of readmissions have estimated proportions of $25.8 \%$ to $53.0 \%$ preventable $^{16}$ and Arozullah $e t a l^{0}$ in their study of general medical admissions deemed $43 \%$ were preventable. Thus, our power calculations based on assumptions of 15-35\% deemed preventable are conservative estimates.

\section{Validation of the PAT}

For the validation of the PAT, if $20 \%$ are deemed preventable, a sample of 150 patients gives power to estimate a $\kappa$ statistic $>0.6 \pm 0.08$; and a sensitivity or specificity of 0.9 with a precision of \pm 0.1 , and $\alpha=0.05$. If more admissions are classified as preventable, the precision is increased.

\section{ETHICS AND DISSEMINATION}

Ethical approval has been obtained from the NSW Population and Health Services Research Ethics Committee. All patients will provide written informed consent prior to any study-related data being collected.

This policy-relevant research has implications for addressing the growth in PPH admissions and the consequent pressure on the health system in Australia and internationally. The findings will help determine both the proportion of chronic PPH admissions that are deemed preventable, and the modifiable drivers contributing to preventable admissions, and will thus help generate an evidence base for the development and targeting of interventions to reduce chronic PPH admissions. Subsequent trials will be developed to test these interventions. More appropriately targeted interventions have the potential to improve the health and quality of life of people with chronic conditions and reduce $\mathrm{PPH}$ admissions.

The project aims to produce a validated tool for prospectively assessing the preventability of individual admissions, enabling identification of chronic $\mathrm{PPH}$ admissions which are considered preventable and the underlying modifiable factors contributing to them, at the time of admission. The validated PAT will be available to researchers and policymakers in other settings, enabling international comparative research and an understanding of how factors that drive preventability are influenced by social and health systems.

With governments facing growing demands on health systems internationally, the research is also of immediate relevance to accountability for taxpayer-funded healthcare, as PPH admissions are used as a performance indicator in many countries. In Australia, the rate of $\mathrm{PPH}$ is a KPI in the $\mathrm{NHA}^{2}$ and the National Health Performance and Accountability Framework ${ }^{3}$ and is therefore tied directly to hospital funding. Our research will inform possible refinements to PPH measures using administrative data that can be applied nationally and internationally to provide more robust performance measurement and may influence policies regarding health service funding.

In order to facilitate dissemination and translation of the findings, we are working closely with a range of policymakers and service providers who provide input through the project Steering Committee. All the funding partners are actively engaged in the Steering Committee, enabling rapid dissemination and discussion of results as they are available. In addition to peerreviewed academic papers, dissemination to a broader audience will include engagement of policy stakeholders through the production of summary reports and presentations for policy and clinical audiences, at state, national and international levels.

Acknowledgements The authors thank the study partners (Mid North Coast Local Health District, Western Sydney Local Health District, North Coast Primary Health Network and the NSW Agency for Clinical Innovation), research nurses, and the staff and management of participating hospitals and General Practices.

Collaborators The DaPPHne investigator team comprises MP, LJ, JL, GM, Lesley Barclay, DE, Vahid Saberi, Sabrina Pit, BC, Stewart Dowrick, JJ and MR.

Contributors MP, LJ, JL, GM and DE had overall responsibility for conceptualisation of this study. MP lead development of original grant application, supported by JL, LJ and MR. MP, JL, LJ, GM, MR, BC, DE and $\mathrm{JJ}$ contributed to the design of the study. JJ drafted this paper with the support of MP and JL, and all contributors approved the final draft.

Funding The DaPPHne study is funded by partner agencies, which at the time of writing, are: the Mid North Coast Local Health District, Western Sydney Local Health District, North Coast Primary Health Network and the NSW Agency for Clinical Innovation.

Competing interests None declared.

Ethics approval NSW Population and Health Services Research Ethics Committee.

Provenance and peer review Not commissioned; internally peer reviewed.

Open Access This is an Open Access article distributed in accordance with the Creative Commons Attribution Non Commercial (CC BY-NC 4.0) license, which permits others to distribute, remix, adapt, build upon this work noncommercially, and license their derivative works on different terms, provided the original work is properly cited and the use is non-commercial. See: http:// creativecommons.org/licenses/by-nc/4.0/

\section{REFERENCES}

1. Billings J, Zeitel L, Lukomnik J, et al. Impact of socioeconomic status on hospital use in New York City. Health Aff (Millwood) 1993;12:162-73. 
2. Council of Australian Governments. National Healthcare Agreement. Secondary National Healthcare Agreement. 2012. http://www. federalfinancialrelations.gov.au/content/npa/healthcare/ natonal-agreement-superseded-Aug11.pdf

3. National Health Performance Authority. Performance and Accountability Framework. Secondary Performance and Accountability Framework. 2012. http://www.nhpa.gov.au/internet/ nhpa/publishing.nst/Content/PAF

4. Australian Institute of Health and Welfare. Australian hospital statistics 2012-13. Health services series no. 50. Cat. no. HSE 145 2014.

5. Jorm LR, Leyland AH, Blyth FM, et al. Assessing Preventable Hospitalisation InDicators (APHID): protocol for a data-linkage study using cohort study and administrative data. BMJ Open 2012;2:pii: e002344.

6. Ansari Z, Laditka JN, Laditka SB. Access to health care and hospitalization for ambulatory care sensitive conditions. Med Care Res Rev 2006;63:719-37.

7. Longman JM, Passey ME, Singer J, et al. The role of social isolation in frequent and/or avoidable hospitalisation: rural community-based service providers' perspectives. Aust Health Rev 2013;37:223-31.

8. Longman JM, Rolfe M, Passey M, et al. Frequent hospital admission of older people with chronic disease: a cross-sectional survey with telephone follow-up and data linkage. BMC Health Serv Res 2012;12:373.

9. Longman J, Singer J, Gao Y, et al. Community based service providers' perspectives on frequent and/or avoidable admission of older people with chronic disease in rural NSW: a qualitative study. BMC Health Serv Res 2011;11:265.

10. Falster MO, Jorm LR, Douglas KA, et al. Sociodemographic and health characteristics, rather than primary care supply, are major drivers of geographic variation in preventable hospitalizations in Australia. Med Care 2015;53:436-45.

11. Feyer A-M, McDonald A, Billot L, et al. State-wide Evaluation. NSW Health Chronic Disease Management Program. Final Report. Sydney: The George Institute for Global Health; The Centre for Primary Health Care and Equity, University of New South Wales; and The Centre for Health Economic Research and Evaluation, University of Technology Sydney, 2014.

12. Page AC, Ambrose SJ, Glover JD, et al. Atlas of Avoidable Hospitalisations in Australia: ambulatory care-sensitive conditions. Adelaide: Public Health Information Development Unit, University of Adelaide, 2007.

13. Banham D, Woollacott T, Gray J, et al. Recognising potential for preventing hospitalisation. Aust Health Rev 2010;34:116.

14. Li SQ, Gray NJ, Guthridge SL, et al. Avoidable hospitalisation in Aboriginal and non-Aboriginal people in the Northern Territory. Med J Aust 2009;190:532-6.

15. Barnett R, Malcolm L. Practice and ethnic variations in avoidable hospital admission rates in Christchurch, New Zealand. Health Place 2010;16:199-208.
16. van Walraven $C$, Bennett $C$, Jennings $A$, et al. Proportion of hospital readmissions deemed avoidable: a systematic review. Can Med Assoc J 2011;183:E391-402

17. Billings $\mathrm{J}$, Dixon J, Mijanovich $\mathrm{T}$, et al. Case finding for patients at risk of readmission to hospital: development of algorithm to identify high risk patients. BMJ 2006;333:327.

18. Longman JM, Passey ME, Ewald DP, et al. Admissions for chronic ambulatory care sensitive conditions - a useful measure of potentially preventable admission? BMC Health Serv Res 2015; 15:472.

19. Oddone EZ, Weinberger $\mathrm{M}$, Horner $\mathrm{M}$, et al. Classifying general medicine readmissions. J Gen Intern Med 1996;11:597-607.

20. Australian Institute of Health and Welfare. Australian hospital statistics 2011-12. Health services series no. 50. Cat. no. HSE 134. Canberra, AlHW, 2013.

21. Ware JE, Kosinski M, Gandek B. SF-36 Health Survey: Manual and Interpretation Guide. Lincoln, RI: QualityMetric Inc., 2000.

22. Kessler RC, Barker PR, Colpe LJ, et al. Screening for serious mental illness in the general population. Arch Gen Psychiatry 2003;60:184-9.

23. Battersby MW, Ask A, Reece MM, et al. The partners in health scale: the development and psychometric properties of a generic assessment scale for chronic condition self-management. Aust $J$ Prim Health 2003;9:41-52.

24. Bass PF, Wilson JF, Griffith $\mathrm{CH}$. A shortened instrument for literacy screening. J Gen Intern Med 2003;18:1036-8.

25. Chew LD, Griffin JM, Partin MR, et al. Validation of screening questions for limited health literacy in a large VA outpatient population. J Gen Intern Med 2008;23:561-6.

26. Koenig HG, Westlund RE, George LK, et al. Abbreviating the Duke Social Support Index for use in chronically ill elderly individuals. Psychosomatics 1993;34:61.

27. Mitchell PH, Powell L, Blumenthal J, et al. A short social support measure for patients recovering from myocardial infarction: the ENRICHD Social Support Inventory. J Cardiopulm Rehabil Prev 2003;23:398-403.

28. Krousel-Wood M, Islam T, Webber LS, et al. New medication adherence scale versus pharmacy fill rates in seniors with hypertension. Am J Manag Care 2009;15:59-66.

29. Pit SW, Byles JE, Cockburn J. Prevalence of self-reported risk factors for medication misadventure among older people in general practice. J Eval Clin Pract 2008:14:203-8.

30. Arozullah AM, Lee SYD, Khan T, et al. The roles of low literacy and social support in predicting the preventability of hospital admission. $J$ Gen Intern Med 2006;21:140-5.

31. Choicemaker[program]. New York: Choicemaker Pty Ltd, 2006

32. Centre for Health Record Linkage. CHeReL Quality Assurance. Secondary CHeReL Quality Assurance. http://www.cherel.org.au/ quality-assurance

33. Braun V, Clarke V. Using thematic analysis in psychology. Qual Res Psychol 2006;3:77-101

34. SAS version 9.3[program]. Cary, NC: SAS Institute, 2011. 